\title{
How many genes...?
}

DOI:

10.1038/nrc2001

\section{URLs}

Links

Breast cancer

http://www.cancer.gov/

cancertopics/types/breast Colorectal cancer

http://www.cancer.gov/

cancertopics/types/colon-

and-rectal
Just how many genes are mutated in a human tumour? A team of researchers working at a number of research institutions in the United States have completed the first unbiased, large-scale sequencing study of protein-coding genes in breast and colorectal cancer. They have identified a surprising number of mutated genes, many of which have not previously been implicated in tumorigenesis.

13,023 verified full-length protein-coding genes from the consensus coding sequences database were initially analysed in 11 cell lines or xenografts of each tumour type. 1,419 genes were found to have somatic mutations. Validation of these results in an additional 24 tumour samples of each tumour type indicated that 189 of these genes are probably candidate cancer (CAN) genes. In addition to the usual sus- pects, such as TP53, KRAS and APC, genes not usually associated with cancer were found to be CAN genes, such as guanylate cyclase 1 and the transcription factor TBX22.

The authors also found that the collection of genes that are mutated differs between breast and colorectal tumours, and that the nature of the mutations that occur in genes that are mutated in both tumour types differ. This supports the long-held view that tissue-specific carcinogenic processes exist.

Interestingly, these results indicate that, on average, each breast tumour has at least 71 protein-altering mutations and that each colorectal tumour has at least 52. If this finding is verified, it means that human tumours have a much higher level of mutation than previously thought. Importantly, the authors conclude, it indicates that our animal and tissue culture models might not reflect the complexity of the human disease.

Nicola McCarthy

ORIGINAL RESEARCH PAPER Sjöblom, T. et al. The consensus coding sequences of human breast and colorectal cancers. Science 7 September 2006 (doi:10.1126/science.1133427)

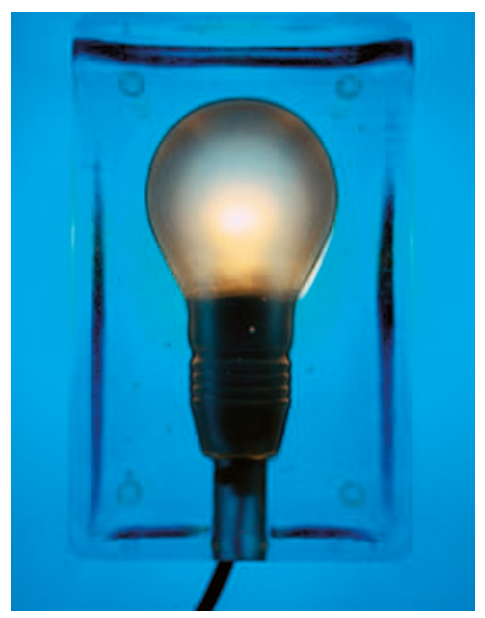

